Pacific Journal of Mathematic 


\title{
A CONVERSE OF HELLY'S THEOREM ON CONVEX SETS
}

\author{
ARYEH DVORETZKY
}

1. Introduction. Helly's well known theorem on convex sets states that families of compact convex sets in Euclidean $n$-space $E^{n}$, have the following property:

Property 14: If every $n+1$ of the sets have a point in common, then there exists a point common to all sets of the family.

If a family of compact sets in $E^{n}$ has property th this, clearly, does not imply that the sets are convex. The purpose of this short note is to show that (loosely speaking) if the family possesses property of not accidentally but by virtue of the geometric structure of its sets, then all the sets of the family are convex. The proof of our result is rather simple, but as far as we are aware no theorems converse to Helly's have been noticed before.

In order to state our result briefly we make the following definition:

DEFINITION. A family of sets $K_{a}$ in $E^{n}$ is said to have property Cid if every family $\left\{K_{a}^{\prime}\right\}$, with $K_{a}^{\prime}=T_{a} K_{a}$ an affine ${ }^{1}$ transform of $K_{a}$, possesses property $d$.

We may now formulate our result.

THEOREM. Let $\left\{K_{a}\right\}$ be a family of more than $n+1$ compact sets in $E^{n}$, all having linear dimension $n$ (that is, no $K_{a}$ lies in a hyperplane). If the family has property $C_{b}$ then all sets $K_{a}$ are convex.

2. Proof. We shall show that if one of the sets of the family, say $K_{0}$, is not convex then the family cannot have property $C_{b}$.

Since $K_{0}$ is closed and its linear dimension is $n$, there exist $n+1$ points $P_{1}, \ldots, P_{n+1} \in K_{0}$ forming the vertices of a simplex whose interior contains points not belonging to $K_{0}$. Let $P_{0}$ be such a point.

\footnotetext{
${ }^{1}$ By an affine transformation we understand a nonsingular one.

Received September 22, 1953.
}

Pacific J. Math. 5 (1955), 345-350 
The family contains more than $n+1$ sets. Let $K_{1}, K_{2}, \cdots, K_{n+1}$ be $n+1$ arbitrary sets of the family different ${ }^{2}$ from $K_{0}$. For $i=1, \cdots, n+1$ let $\hat{K}_{i}$ denote the convex hull of $K_{i}$. Let $Q_{0}^{(i)}$ be any extreme point of $\hat{K}_{i}$ (that is, $Q_{0}^{(i)} \in \hat{K}_{i}$ and it is not an interior point of any segment contained in $\hat{K}_{i}$ ). Since $K_{i}$ is compact and not empty there exists such a point and, moreover, $Q_{0}^{(i)} \in K_{i}$. Let $\pi_{i}$ be a hyperplane containing $Q_{0}^{(i)}$ and such that all other points of $\hat{K}_{i}$ are in one of the open half-spaces, say $\pi_{i}^{+}$, determined by it (such a plane $\pi_{i}$ exists since $Q_{0}^{(i)}$ is an extreme point of $\hat{K}_{i}$ ). As $K_{i}$ has linear dimension $n$ there exist points $Q_{1}^{(i)}, \ldots, Q_{n}^{(i)} \in K_{i}$, such that the $n+1$ points $Q_{0}^{(i)}, Q_{1}^{(i)}, \ldots, Q_{n}^{(i)}$ form the vertices of a simplex. Let $T_{i}$ be an affine transformation sending $Q_{0}^{(i)}$ into $P_{0}$ and $Q_{1}^{(i)}, \ldots, Q_{n}^{(i)}$ into the $n$ points $P_{j}$ with $1 \leq j \leq n+1$ and $j \neq i$.

Let $T_{0}$ denote the identity transformation. Also, if the family contains more then $n+2$ sets, associate with every set $K_{\beta}$, different from the $n+2$ sets already considered, an affine transformation $T_{\beta}$ such that $T_{\beta} K_{\beta}$ contains the $n+1$ points $P_{1}, \cdots, P_{n+1}$ (this is possible since the linear dimension of $K_{\beta}$ is $n)$.

Put $K_{\alpha}^{\prime}=T_{\alpha} K_{\alpha}$ (for $K_{a}=K_{i}, i=0,1, \cdots, n+1$ as well as for $\left.K_{\beta}=K_{\alpha}\right)$. Now every $n+1$ of the sets $K_{\alpha}^{\prime}$ have a point in common. Indeed, the sets $K_{1}^{\prime}, \cdots, K_{n+1}^{\prime}$ have the point $P_{0}$ in common, while any other collection of $n+1$ sets $K_{a}^{\prime}$ must omit at least one of these sets, say $K_{i}^{\prime}$ and then $P_{i}$ belongs to all the $K_{\alpha}^{\prime}$ in the collection. On the other hand we shall prove that there is no point common to all $K_{a}^{\prime}$. This will be done by showing that $\bigcap_{i=1}^{n+1} K_{i}^{\prime}=\phi$ (the void set).

This last assertion is established as follows. (i) Since

$$
K_{i} \subset Q_{i}^{(0)} \cup \pi_{i}^{+}
$$

we have

$$
K_{i}^{\prime} \subset P_{0} \cup T_{i} \pi_{i}^{+}
$$

for $i=1, \cdots, n+1$, and, therefore,

$$
\bigcap_{i=1}^{n+1} K_{i}^{\prime} \subset P_{0} \cup\left(\bigcap_{i=1}^{n+1} T_{i} \pi_{i}^{+}\right)
$$

${ }^{2}$ Different means labelled differently. The theorem applies also to families in which one set appears several times, for example, to a family consisting of $n+2$ identical sets. 
(ii) For $i=1, \cdots, n+1$ let $C_{i}$ denote the closed polyhedral cone with vertex at $P_{0}$ and edges obtained by prolongation of the $n$ directed segments ${\overrightarrow{P_{j}}}_{P_{0}}$ $(1 \leq j \leq n+1, j \neq i)$. Since

$$
P_{j} \in K_{i}^{\prime} \cap T_{i} \pi_{i}^{+}
$$

for these $j$, we have $C_{i} \cap T_{i} \pi_{i}^{+}=\phi$; also, since $P_{0}$ is an interior point of the simplex with vertices $P_{1}, \ldots, P_{n+1}$, we have $U_{i=1}^{n+1} C_{i}=E^{n}$, the whole space. Therefore

$$
\bigcap_{i=1}^{n+1} T_{i} \pi_{i}^{+}=\bigcup_{i=1}^{n+1}\left(C_{i} \cap\left(\bigcap_{i=1}^{n+1} T_{i} \pi_{i}^{+}\right)\right) \subset \bigcup_{i=1}^{n+1}\left(C_{i} \cap T_{i} \pi_{i}^{+}\right)=\phi
$$

(iii) Combining this with the result of (i) we have

$$
\bigcap_{i=1}^{n+1} K_{i}^{\prime} \subset P_{0}
$$

Thus $P_{0}$ is the only common point of $K_{1}^{\prime}, \cdots, K_{n+1}^{\prime}$, but $P_{0} \notin K_{0}^{\prime}=K_{0}$, hence there is no common point to the $n+2$ sets $K_{0}^{\prime}, K_{1}^{\prime}, \cdots, K_{n+1}^{\prime}$. Q.e.d.

3. Generalizations. We indicate two stronger versions of the theorem of $\S 1$.

3.1. Similarly to the way we defined property Cidf, we can define the weaker property $a^{+}+d$ by restricting the affine transformations $T_{a}$ in the definition, to those for which the determinant of the non-translational part is positive. Only minor modifications are required in the proof in order to show that the theorem of $\S 1$ remains valid if property $C_{0} \&$ is replaced by property $a^{+} \not \psi_{\text {. }}$

3.2. Let $K$ be a closed set in $E^{n}$ having linear dimension $n$, and such that its complement in $E^{n}$ contains a nondegenerate cone. It can then be shown that there exist points $Q_{0}, Q_{1}, \cdots, Q_{n} \in K$ forming the vertices of a simplex, and having the further property that $Q_{0}$ is the only point belonging to $K$ in the closed cone having $Q_{0}$ as vertex and whose edges are the prolongations of $\overrightarrow{Q_{i} Q_{0}}$, $i=1, \cdots, n$. Using this fact the proof of $\$ 2$ easily yields the theorem of $\$ 1$ with the assumption of compactness weakened to: every $K_{a}$ is closed and its complement contains a nondegenerate cone.

3.3. For $n \geq 2$ both 3.1 and 3.2 can be carried out simultaneously. That this cannot be done for $n=1$ is shown by the following example: A family of 
3 sets, one consisting of two points and the other two being two equally directed closed half lines.

4. Remarks. It might be interesting to consider the necessity of the various assumptions made in the theorem.

4.1. It is natural to ask whether property Cid could be weakened in that we would allow not all affine transformations but only some transformations of a special kind. As shown in 3.1 it is possible to do something in this direction; however not much more can be done as is seen from examples that follow.

The theorem would become false if in defining property Cidf we would have restricted the affine transformations by the extra condition that the determinant of the nontranslational part of the transformation be rational. Indeed, let the family contain $n+2$ sets $S_{1}, \ldots, S_{n+2}$, each $S_{i}$ consisting of $n+1$ points forming the vertices of a simplex. Let $V_{i}$ be the volume of the simplex whose set of vertices is $S_{i}$ and assume the numbers $V_{1}, V_{2}, \ldots, V_{n+2}$ to be rationally independent. We claim that $S_{i}^{\prime}=T_{i} S_{i},(i=1, \cdots, n+2)$ with $T_{i}$ being affine

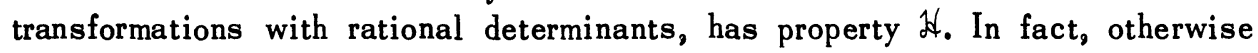
we would have $\cap_{i=1}^{n+2} S_{i}^{\prime}=\phi$ while any $n+1$ of the sets $S_{i}^{\prime}$ would have a point in common. This would be possible only if $\cup_{i=1}^{n+2} S_{i}^{\prime}$ consists of exactly $n+2$ points, and the $n+2$ sets $S_{i}^{\prime}$ are all the different subsets of $n+1$ points of $\cup_{i=1}^{n+2} S_{i}^{\prime}$. We may denote the points by $Q_{1}, Q_{2}, \ldots, Q_{n+2}$ in such a way that $S_{i}^{\prime}$ consists of all these points except $Q_{i}$. Let $v_{i}$ be the volume of $S_{i}$ then either (i) one $v_{i}$ is equal to the sum of the $n+1$ numbers $v_{j}$ with $i \neq j$ (this happens if one of the points $Q_{1}, \cdots, Q_{n+2}$ is an interior point of the simplex formed by the other points); or (ii) the sum $v_{i}+v_{j}$ of two volumes equals the sum of two of the $n$ remaining $v_{k}$, (this happens if the previous case does not occur). But $v_{i}=\left|d_{i}\right| V_{i}(i=1, \cdots, n+2)$ where $d_{i}$ is the determinant (of the nontranslational part) of $T_{i}$, and either (i) or (ii) would imply a rational relation between the $V_{i}$ contrary to our assumption.

An argument of the same kind shows the existence of $n+2$ sets $S_{i}$ as above having the property obtained from $C$ fof by restricting the affine transformations by the condition that the determinants be bounded and bounded away from zero. Such an argument also applies if instead of considering all affine transformations we consider, say, the similarities, that is, those obtained by combinations of translations, stretchings and orthogonal transformations; etc.

It should be noticed that in the above counterexamples the families consist of $n+2$ sets; thus they apply even if in the theorem the assumption "the family 
has property Cad" is strengthened to "every subfamily of more than $n+1$ sets has property GJf". On the other hand it is easily seen that with this new formulation (but not with the original one) the theorem remains valid if we restrict the consideration to affine transformations with determinants bounded by an arbitrary positive number (or, alternatively, with determinants bounded away from zero).

4.2. In 3.2 we remarked that the assumption of compactness could be weakened; it is, however, impossible to dispense with it altogether. To see this let $O_{i}(i=1,2, \cdots, N, N>n+1)$ be nonvoid, open and convex sets in $E^{n}$. Let $O_{i}^{*}$ be a set obtained from $O_{i}$ by deleting a single point $P_{i}$ from it. The sets $O_{i}^{*}$ are not convex, yet we claim that the family consisting of these $N$ sets has property Cizf. Indeed, let $T_{i}(i=1, \cdots, N)$ be affine transformations. If every $n+1$ of the sets $T_{i} O_{i}^{*}$ have a point in common, so do a fortiori every $n+1$ of the sets $T_{i} O_{i}$. But the sets $T_{i} O_{i}(i \neq 1, \cdots, N)$ are convex and, it is well

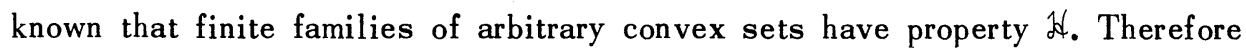
$\bigcap_{i=1}^{N} T_{i} O_{i} \neq \phi$, but $\bigcap_{i=1}^{N} T_{i} O_{i}$ is an open set, hence it must contain other points besides $T_{i} P_{i}(i=1, \cdots, N)$. Since

$$
\bigcap_{i=1}^{N} T_{i} O_{i} \subset\left(\bigcap_{i=1}^{N} T_{i} O_{i}^{*}\right) \cup\left(\bigcup_{i=1}^{N} T_{i} P_{i}\right)
$$

it follows that $\bigcap_{i=1}^{N} T_{i} O_{i}^{*} \neq \phi$, that is, our family has property $C_{0} \alpha_{\text {as }}$ claimed.

It is even impossible, unless some precautions are taken, to replace the word "compact" in the theorem by the word "closed". One has merely to think of the family $\left\{K_{1}, \ldots, K_{n+2}\right\}$ where $K_{1}, \ldots, K_{n+1}$ are arbitrary sets of linear dimension $n$ and $K_{n+2}=E^{n}$.

4.3. Finally, it is easy to see that the assumption about the linear dimension of the sets $K_{\alpha}$ is essential. The simplest example proving this is obtained by considering the case when each $K_{a}$ consists of $n$ (or fewer) points. The sets $K_{a}^{\prime}$ consist also of fewer than $n+1$ points and a trivial argument shows that the family $\left\{K_{a}^{\prime}\right\}$ has property df.

It is also impossible to improve the theorem by dropping the assumption about the linear dimension and replacing the conclusion by "each set is either convex or has linear dimension n". A trivial counterexample is obtained by taking one arbitrary set and all other sets consisting of single points. It is possible to construct more ingenious examples showing, for example, that one 
cannot replace the assumption that the linear dimension is $n$ by the assumption that the sets contain more than $n$ points.

HEBREW UNIVERSITY, JER USALEM . 


\section{PACIFIC JOURNAL OF MATHEMATICS}

\section{EDITORS}

\author{
H.L. ROYDEN \\ Stanford University \\ Stanford, California \\ E. HEWITT \\ University of Washington \\ Seattle 5, Washington
}

\author{
R. P. DILWORTH \\ California Institute of Technology \\ Pasadena 4, California \\ * Alfred Horn \\ University of California \\ Los Angeles 24, California
}

\section{ASSOCIATE EDITORS}

\section{H, BUSEMANN \\ HERBERT FEDERER}

MARSHALL HALL

\section{P.R. HALMOS \\ HEINZ HOPF}

ALFRED HORN

\author{
R.D. JAMES \\ BØRGE JESSEN \\ PAUL LÉVY
}

GEORGE PÓLYA

J.J. STOKER

KOSAKU YOSIDA

\section{SPONSORS}

UNIVERSITY OF BRITISH COLUMBIA

UNIVERSITY OF SOUTHERN CALIFORNIA

CALIFORNIA INSTITUTE OF TECHNOLOGY

UNIVERSITY OF CALIFORNIA, BER KELEY

STANFORD RESEARCH INSTITUTE

STANFORD UNIVERSITY

UNIVERSITY OF CALIFORNIA, DAVIS

UNIVERSITY OF. UTAH

UNIVERSITY OF CALIFORNIA, LOS ANGELES

WASHINGTON STATE COLLEGE

UNIVERSITY OF CALIFORNIA, SANTA BARBARA

UNIVERSITY OF WASHINGTON

MONTANA STATE UNIVERSITY

UNIVERSITY OF NEVADA

OREGON STATE COLLEGE

AMERICAN MATHEMATICAL SOCIETY

HUGHES AIRCRAFT COMPANY

UNIVERSITY OF OREGON

SHELL DEVELOPMENT COMPANY

\section{UNIVERSTTY OF SOUTHERN CALIFORNIA}

Mathematical papers intended for publication in the Pacific Journal of Mathematics should be typewritten (double spaced), and the author should keep a complete copy. Manuscripts may be sent to any of the editors. Manuscripts intended for the outgoing editors should be sent to their successors. All other communications to the editors should be addressed to the managing editor, Alfred Horn, at the University of California Los Angeles 24, California.

50 reprints of each article are furnished free of charge; additional copies may be obtained at cost in multiples of 50 .

The Pacific Journal of Mathematics is published quarterly, in March, June, September, and December. The price per volume (4 numbers) is $\$ 12.00$; single issues, $\$ 3.50$; back numbers (Volumes $1,2,3$ ) are available at $\$ 2.50$ per copy. Special price to individual faculty members of supporting institutions and to individual members of the American Mathematical Society: $\$ 4.00$ per volume; single issues, $\$ 1.25$.

Subscriptions, orders for back numbers, and changes of address should be sent to the publishers, University of California Press, Berkeley 4, California.

Printed at Ann Arbor, Michigan. Entered as second class matter at the Post Office, Berkeley, California.

* During the absence of E.G. Straus.

UNIVERSITY OF CALIFORNIA PRESS - BERKELEY AND LOS ANGELES 


\section{Pacific Journal of Mathematics}

\section{Vol. 5, No. $3 \quad$ November, 1955}

Nesmith Cornett Ankeny and S. Chowla, On the divisibility of the class number of quadratic fields ............................. 321

Cecil Edmund Burgess, Collections and sequences of continua in the plane ........................................ 325

Jane Smiley Cronin Scanlon, The Dirichlet problem for nonlinear elliptic equations....................................... 335

Arieh Dvoretzky, A converse of Helly's theorem on convex sets ......... 345

Branko Grünbaum, On a theorem of L. A. Santaló................ 351

Moshe Shimrat, Simple proof of a theorem of P. Kirchberger .......... 361

Michael Oser Rabin, A note on Helly's theorem . ................... 363

Robert E. Edwards, On factor functions . . ................... 367

Robert E. Edwards, On certain algebras of measures ............... 379

Harley M. Flanders, Methods in affine connection theory.............. 391

Alfred Huber, The reflection principle for polyharmonic functions ........ 433

Geoffrey Stuart Stephen Ludford, Generalised Riemann invariants ....... 441

Ralph Gordon Selfridge, Generalized Walsh transforms............. 451 